Original Research Paper

\title{
Procalcitonin and Clinical Factors Associated with Severe Dengue Infection in Hospitalized Adults in Malaysia
}

\author{
${ }^{1}$ Huzairi Sani, ${ }^{2}$ Nada Syazana, ${ }^{3}$ Zahir Izuan, ${ }^{3}$ Mohamad Rodi Isa, ${ }^{1}$ Muhammad Hanis and ${ }^{1}$ Muhamad Yazli \\ ${ }^{1}$ Internal Medicine, Universiti Teknologi MARA, Sungai Buloh, Malaysia \\ ${ }^{2}$ Pathology, Ministry of Health, Malaysia \\ ${ }^{3}$ Public health, Universiti Teknologi MARA, Sungai Buloh, Malaysia
}

\author{
Article history \\ Received: 29-07-2018 \\ Revised: 20-11-2019 \\ Accepted: 03-12-2019 \\ Corresponding Author: \\ Huzairi Sani \\ Internal Medicine, Universiti \\ Teknologi MARA, Sungai \\ Buloh, Malaysia \\ Email: huzairis@gmail.com
}

\begin{abstract}
Dengue Fever (DF) is the commonest mosquito-borne viral infection that results in hospitalisation amongst patients in Malaysia. The cornerstone of managing dengue virus infection is the prediction hence prevention of severe DF from developing. Warning signs proposed by WHO as clinical predictors of severe DF are insensitive. Procalcitonin (PCT) has been shown to increase in bacterial infections and is useful in predicting disease severity and mortality. Objective: To determine the level of PCT and other clinical and biochemical parameters associated with severe DF. This is a crosssectional study conducted over 6 months from September 2017 to February 2018. Patients aged 18 years and above who were hospitalised in Hospital Selayang for a serologically-confirmed DF were recruited. PCT level was taken within $24 \mathrm{~h}$ of hospital admission and patients were followed up throughout admission until discharge or death. Out of 133 recruits, $117(88 \%)$ had uncomplicated DF and $16(12 \%)$ had severe DF either with shock $(44 \%)$ or organ failure $(56 \%)$. There were $2(13 \%)$ deaths from the severe group. Median PCT levels were higher in severe [0.35 ng/mL (0.15-4.4)] versus non-severe $[0.28 \mathrm{ng} / \mathrm{mL}(0.17-0.54)] \mathrm{DF}$, however there was no significant difference between both groups $(p=0.518)$. Other significant clinical and biochemical factors associated with severe DF were defervescence phase $(p=0.043)$, lethargy $(p=0.000)$ and albumin $<35 \mathrm{~g} / \mathrm{L}(p=0.015)$. On multivariate analysis, parameters significantly associated with severe DF include lethargy $(p=0.001)$ and albumin $<35 \mathrm{~g} / \mathrm{L}(p=0.009)$. These two parameters plus PCT of $>0.3 \mathrm{ng} / \mathrm{mL}$ predict severe DF with a sensitivity of $73 \%$ and specificity of $85 \%$. PCT [median $3.6 \mathrm{ng} / \mathrm{mL}(3.2-4.0)$ ] is also significantly associated with death $(p=0.021)$. PCT at a cut-off of $>0.3 \mathrm{ng} / \mathrm{mL}$ predicts severe DF when combined with lethargy and albumin $<35 \mathrm{~g} / \mathrm{L}$. Furthermore, PCT is significantly associated with death.
\end{abstract}

Keywords: Dengue, Fever, Severe Dengue, Procalcitonin

\section{Introduction}

Dengue Fever (DF) is one of the most important arthropod-borne viral diseases owing to its high morbidity and mortality. In 2015, the World Health Organization (WHO) of Western Pacific Region (WPRO) reported DF as the fastest emerging arboviral infection. About half of the world's population is now at risk of the infection. Mortality of DF can be as high as $20 \%$ if untreated, whereas if recognised earlier and managed appropriately, it is less than 1\% (Simmons et al., 2012; Lin et al., 2013).
In Malaysia, Dengue fever is a serious public health concern with an escalating trend of infection. From the year 2000 to 2010 , the average number of DF cases and death tolls per annum had recorded a surge of $14 \%$ and $8 \%$ respectively (Mia et al., 2013). Since the year 2000, its incidence in Malaysia continued to increase from 32 cases per 100,000 populations to 328.3 cases per 100,000 populations in 2016 , resulting in a 10 -fold surge in incidence rate in 16 years. According to the Crisis Preparedness and Response Centre (CPRC) of Ministry of Health 
Malaysia, 1,034 DF cases were reported in the first week of 2018 alone. Selangor, Johor and Pulau Pinang had the highest statistics with 490, 140 and 95 reported cases respectively (CPG Malaysia, 2018).

In 1995, a total of 28 DF deaths were reported. The death toll increased by 12 fold to 336 deaths in 2015 , making it the highest fatality rate in the last 20 years. In 2016, the number of deaths dropped to 237 out of 101,357 cases of DF (CPG Malaysia, 2016).

Irrespective of age group, Malaysians are at risk of DF due to its hyperendemicity. Delay in treatment in DF is life threatening. Thus, early recognition of symptoms, early diagnosis and immediate care are required in reducing the risk of developing severe DF. In current clinical practice, there is no reliable clinical and biochemical parameter used to predict severe DF. Plasma lactate is frequently used as a monitoring marker in suspected severe cases but its sensitivity and specificity in predicting disease progression and outcome is poor (Bur et al., 2016).

Recently, the Sequential Organ Failure Assessment (SOFA) score has replaced SIRS in classifying patients with sepsis. According to the Third International Consensus Definitions for Sepsis and Septic Shock (Sepsis-3), sepsis is defined as a life-threatening organ dysfunction caused by dysregulated host-response to systemic infection (Singer et al., 2016).

Procalcitonin (PCT) is produced, expressed and released into the bloodstream as part of the inflammatory response to infection, inflammation or injuries in various tissues (Becker et al., 2004; Linscheid et al., 2004), making it a promising diagnostic and prognostic tool in defining patients with sepsis (SIRS) (Giamarellos-Bourboulis et al., 2004; Novotny et al., 2007). A recent study has also shown PCT to be a good tool in diagnosing and prognosticating sepsis when coupled with SOFA scores (Spoto et al., 2018).

Having a biomarker that predicts severe DF is highly important as maximal treatment can be instated promptly to prevent disease progression. This treatment includes early admission to the Intensive Care Unit or High Dependency Ward for close monitoring and treatment optimisation. This may substantially reduce morbidity and mortality rates of severe Dengue fever.

\section{Materials and Methods}

\section{Procalcitonin}

Procalcitonin (PCT) is a precursor peptide for the hormone calcitonin and was first known to medical literature in (Moya et al., 1975). It is a 116-amino acid peptide with a molecular weight of $14.5 \mathrm{kDa}$, consisting of 3 sections - the amino terminus (57 amino acids), immature calcitonin (33 amino acids) and calcitonin carboxyl-terminus peptide 1 (CCP-1) also known as katacalcin (21 amino acids). Its production is governed by the calcitonin 1 gene (CALC-1) on chromosome 11. The product of this gene, pre-PCT, undergoes proteolytic cleavage producing PCT, which is further processed to the mature calcitonin molecule.

Several studies have proven PCT to be superior to tumor necrosis factor alpha, interleukin-6 and Creactive protein in prognosticating critically ill patients (de Werra et al., 1997; Oberhoffer et al., 1999). The only one-to-one relationship study looking at serum procalcitonin in DF was conducted in Mahidol University Bangkok, Thailand in 2013-2015. This study demonstrated that a PCT level of $>0.7 \mathrm{ng} / \mathrm{mL}$ is independently associated with Dengue shock and/or organ failure (Thanachartwet et al., 2016). Another study in Taiwan found that at a cut-off value of 1.14 $\mathrm{ng} / \mathrm{mL}, \mathrm{PCT}$ has a sensitivity and specificity of $81.5 \%$ and $59.5 \%$ respectively in detecting severe DF with concomitant bacteremia in the ICU (Chen et al., 2016).

PCT was determined by Procalcitonin Rapid Test Kit (EasyDiagnosis Biomedicine Co., Ltd, Wuhan, China) according to the manufacturer's instructions. $0.5 \mu \mathrm{L}$ of whole blood sample was used for PCT analysis. The detection limit for the PCT assay was $0.1 \mathrm{ng} / \mathrm{mL}$ and its coefficients of variation for low and high concentrations were $5.9 \%$ and $6.9 \%$, respectively.

\section{Dengue Warning Signs}

In Dengue fever, warning signs include abdominal pain or tenderness, persistent vomiting, mucosal bleed, clinical fluid accumulation, lethargy, restlessness, liver enlargement of $>2 \mathrm{~cm}$ and increase in haematocrit with concurrent rapid decrease in platelet counts. These warning signs are used as one of the criteria for hospital admission for observation and management of DF as per the 2009 WHO guidelines (WHO, 2009).

Leo et al. (2013) conducted a prospective cohort study from 2010 to 2012 to identify the sensitivity and specificity of warning signs in predicting disease progression. From 499 patients with confirmed Dengue fever, of those who progressed to severe illness, $16.3 \%$ had warning signs on the same day while $51.3 \%$ had warning signs the day before developing severe illness. Out of the list of warning signs, there is no single warning sign that independently predicted disease progression (Leo et al., 2013).

This study is a cross-sectional study conducted in Selayang Hospital, Selangor, Malaysia from September 2017 to February 2018. Patients aged 18 years and above, admitted to Selayang Hospital for acute febrile illness with a positive Dengue virus serology test were recruited. Inclusion criteria were (i) patients aged 18 years and above (ii) confirmed DF by NS-1 antigen or 
IgM serology tests (iii) fever less than 10 days and (iv) patients with warning signs admitted to Selayang Hospital. The exclusion criteria include (i) patients with recent trauma or fall (ii) patients who had undergone a recent surgery (iii) patients with acute or chronic pancreatitis (iv) patients diagnosed with malignancy (NET, MTC, carcinoid tumor, small cell lung cancer) and (v) patients with obvious source of other infections e.g., abscess, cellulitis, pneumonia, meningitis.

Patients were clerked and examined on the day of admission. Investigators were not involved in the management of the subjects in any way. All patients received standard management according to the 2015 Malaysian guidelines "Management of Dengue Infection in Adults". Subjects were merely observed in terms of clinical progression and biochemical changes; and followed up until day of discharge. Severity and final outcome of DF were extracted from each subject's discharge summary. Subjects were only involved during the hospital stay with no further follow up or clinic appointments following discharge.

Plasma PCT was measured on the day of admission. The treating medical team was blinded to the PCT results. $2 \mathrm{ml}$ of blood was sampled into a citrate tube and analysed on the eDiagnosis-Immune Quantitative Analyser QMT-8000. This analyser is a 5-channel tests analyser that allows different tests to be run in parallel.

\section{Results}

About 260 suspected DF cases were admitted to Selayang Hospital under the department of Internal Medicine from September 2017 to February 2018. Out of the 260 cases, 127 cases were excluded due to negative serology testing on admission $(n=67,52.8 \%)$, presence of co-infection $(\mathrm{n}=44,34.6 \%)$ and being below 18 years of age $(n=16,12.6 \%)$. Of the 133 subjects recruited, $117(88 \%)$ had non-severe DF with warning signs and $16(12 \%)$ had severe DF with shock and/or organ failure. The 16 severe cases further saw $7(44 \%)$ subjects with severe plasma leakage and 9 $(56 \%)$ with severe organ impairment - the latter encompassing $5(31.3 \%)$ cases of acute kidney injury, $2(12.5 \%)$ myocarditis, $1(6.3 \%)$ encephalitis and 1 (6.3\%) hepatitis with AST of $>1000$ IU/L. Also amongst the severe DF subjects, 7 (43.8\%) had shock and $1(6.3 \%)$ had fluid accumulation with respiratory distress. There were $2(1.5 \%)$ deaths from complications of DF in this study cohort (Fig. 1).

The cohort's median age was 30 (IQR 23-46) years old. $75(56 \%)$ subjects were male and the remaining 58 (43.6\%) were female. Out of these female patients, 4 were pregnant (6.9\%). Recruited subjects were multiracial, consisting of 74 Malays (55.2\%), 26
Indians (19.4\%), 23 Chinese (17.2\%), 8 Aborigines $(6 \%)$ and 3 non-Malaysians (2.2\%) (Table 1). Subjects were admitted on median day 5 (IQR 4-6) of illness in the febrile phase [median temperature of $38.2^{\circ} \mathrm{C}$ (IQR 37.6-38.8)] and the median duration of hospital stay was 4 (IQR 3-5) days. All subjects presented with warning signs with the most common sign being vomiting $(\mathrm{n}=67,50.4 \%)$ followed by diarrhoea $(\mathrm{n}=$ $66,49.6 \%)$, thrombocytopenia with raised hematocrit (n $=61,45.9 \%)$, abdominal pain $(n=32,24.1 \%)$, lethargy $(\mathrm{n}=16,12 \%)$, mucosal bleeds $(\mathrm{n}=12,9 \%)$, tender hepatomegaly $(\mathrm{n}=2,1.5 \%)$ and clinical fluid accumulation $(\mathrm{n}=1,0.8 \%) .69(52 \%)$ subjects were haemodynamically stable on admission except for 8 (6\%) subjects who presented with decompensated shock.

Cohorts presented with leucopenia (median WCC $3.3 \times 10^{9}$ cells/L, IQR 2.3-5.2), within range albumin (median albumin $38 \mathrm{~g} / \mathrm{L}$, IQR 34-41) and mild aspartate transaminitis (median AST 93 IU/L, IQR 55183). Plasma lactate and plasma procalcitonin were raised in this cohort with a median value of 1.63 $\mathrm{mmol} / \mathrm{L}$ (IQR 1.21-2.22) and $0.28 \mathrm{ng} / \mathrm{ml}$ (IQR 0.17$0.62)$ respectively.

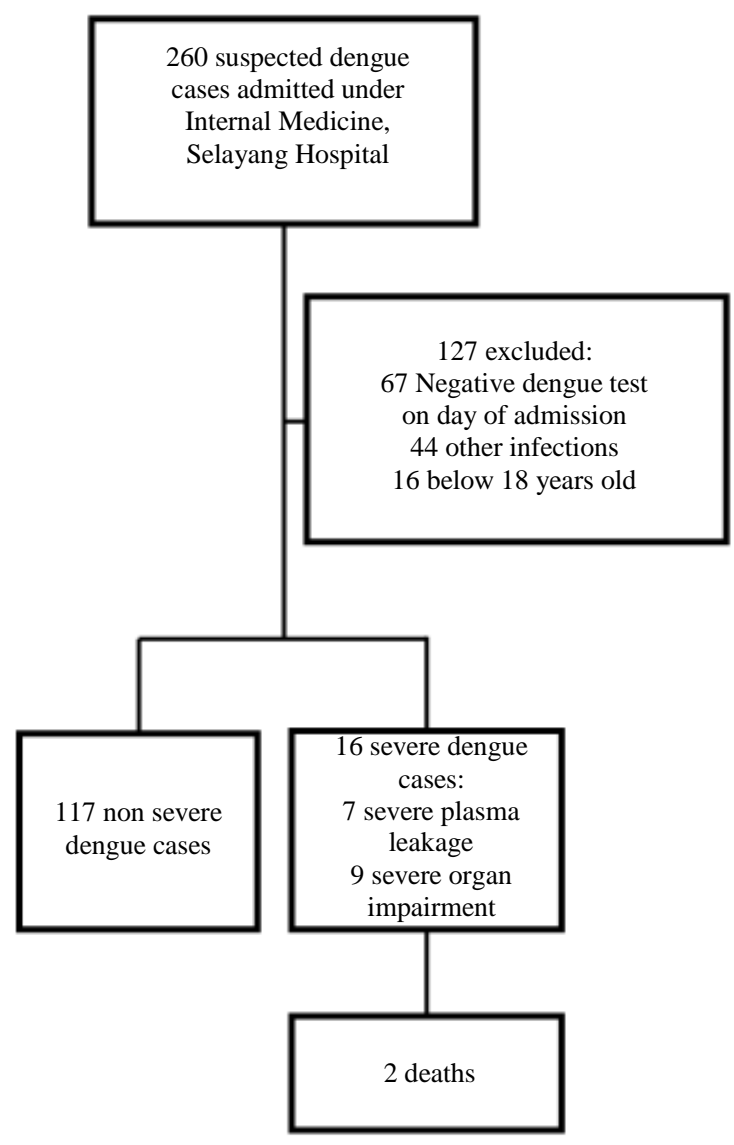

Fig. 1: Flow diagram of recruits and outcome 
Table 1: Demographics and baseline characteristics on day of admission

\begin{tabular}{|c|c|}
\hline Baseline characteristics & Descriptive statistic \\
\hline Age, years & $30(23-46)$ \\
\hline \multicolumn{2}{|l|}{ Gender } \\
\hline Male & $75(56.4 \%)$ \\
\hline Female & $58(43.6 \%)$ \\
\hline \multicolumn{2}{|l|}{ Race } \\
\hline Malay & $74(56.1 \%)$ \\
\hline Indian & $26(19.7 \%)$ \\
\hline Chinese & $23(17.4 \%)$ \\
\hline Aborigines & $8(6.1 \%)$ \\
\hline Bangladeshi & $2(1.6 \%)$ \\
\hline Nepalese & $1(0.8 \%)$ \\
\hline Pregnant & $4(6.9 \%)$ \\
\hline Day of illness & $5(3-6)$ \\
\hline Days of admission & $4(3-5)$ \\
\hline \multicolumn{2}{|l|}{ Warning signs } \\
\hline Vomiting & $67(50.4 \%)$ \\
\hline Diarrhoea & $66(49.6 \%)$ \\
\hline Raised haematocrit with low platelet & $61(45.9 \%)$ \\
\hline Abdominal pain & $32(24.1 \%)$ \\
\hline Lethargy & $16(12 \%)$ \\
\hline Mucosal bleeding & $12(9 \%)$ \\
\hline Tender hepatomegaly & $2(1.5 \%)$ \\
\hline Clinical fluid accumulation & $1(0.8 \%)$ \\
\hline \multicolumn{2}{|l|}{ Co-morbidity } \\
\hline Diabetes mellitus & $11(8.3 \%)$ \\
\hline Hypertension & $11(8.3 \%)$ \\
\hline \multicolumn{2}{|l|}{ Vital signs } \\
\hline Decompensated shock $(\%)$ & $8(6 \%)$ \\
\hline Pulse rate $>90$ beats per minute & $100(95-105)$ \\
\hline Pulse rate $<90$ beats per minute & $76(72-84)$ \\
\hline Febrile phase, $(\%)$ & $88(66 \%)$ \\
\hline Defervescence phase, $(\%)$ & $45(34 \%)$ \\
\hline Respiratory rate per minute & $20(18.5-20)$ \\
\hline \multicolumn{2}{|l|}{ Blood parameters } \\
\hline Haemoglobin, g/dL & $14.3(13.1-16.0)$ \\
\hline Platelet x 109 cells $/ \mathrm{L}$ & $80(36-132)$ \\
\hline Haematocrit $\%$ & $42(39-46)$ \\
\hline Urea, mmol/L & $3.3(2.6-4.6)$ \\
\hline Sodium, mmol/L & $133(131-135)$ \\
\hline Potassium, mmol/L & $3.6(3.2-3.9)$ \\
\hline Creatinine, $\mu \mathrm{mol} / \mathrm{L}$ & $71(56-90)$ \\
\hline
\end{tabular}

Variables are described as median (IQR) interquartile range and frequency $(\%)$ percentage

Table 2: Median values of biomarkers

\begin{tabular}{ll}
\hline Baseline characteristics & Descriptive statistics \\
\hline White blood cell x 109cells/L & $3.3(2.3-5.2)$ \\
Albumin, g/L & $38(34-41)$ \\
AST, IU/L & $93(55-183)$ \\
Lactate, $\mathrm{mmol} / \mathrm{L}$ & $1.63(1.21-2.22)$ \\
Procalcitonin, $\mathrm{ng} / \mathrm{ml}$ & $0.28(0.17-0.62)$ \\
\hline Variables are described as median (IQR)
\end{tabular}

Severe DF patients presented in afebrile phase or critical phase compared to the non-severe group ( $p=$ $0.043)$. The severe group was also likely to present with lethargy $(p=0.00)$ and had a lower median systolic blood pressure of $94 \mathrm{mmHg}(p=0.000)$ and diastolic blood pressure of $56 \mathrm{mmHg}(p=0.000)$ as compared to the non-severe group (systolic blood pressure 120 $\mathrm{mmHg}$ and diastolic blood pressure $76 \mathrm{mmHg}$ ). A full breakdown of clinical variables are shown in Table 3 .

About 5 laboratory parameters assessed in severe DF were analysed namely procalcitonin, WCC, plasma albumin, AST and lactate. Plasma procalcitonin level were raised in both groups with a median of $0.28 \mathrm{ng} / \mathrm{ml}$ (IQR 0.17-0.62). These levels were higher in the severe group with a median of 0.35 $\mathrm{ng} / \mathrm{mL}$ (IQR 0.15-4.4) in comparison to the nonsevere group's median value of 0.28 (IQR 0.17-0.54) but with an insignificant $p$-value of 0.518 . Median values of raw data concerning the other 4 biomarkers also showed non-significant differences between severe and non-severe DF groups (Table 2).

Cut-offs for WCC and PCT were determined based on the level with best specificity and sensitivity by ROC curve analysis. The cut-off level for WCC was $>3 \times 10^{9} / \mathrm{L}$ (specificity $58 \%$, sensitivity $50 \%$ ) and PCT was $>0.3$ $\mathrm{ng} / \mathrm{mL}$ (specificity $50 \%$, sensitivity $64 \%$ ).

The remaining 3 parameters failed to generate a good ROC curve at any cut-off, hence the normal limit of each parameter was defined as the cut-off level. Univariate analysis of these parameters showed that plasma albumin of $<35 \mathrm{~g} / \mathrm{L}$ was significantly associated with severe DF $(p=0.015)$. All other parameters did not show significant associations with either group.

\section{Multivariate Analysis}

Multi-linear regression was done on multiple variables including defervescence phase, lethargy, albumin of $<35 \mathrm{~g} / \mathrm{L}$ and plasma procalcitonin of $>0.3$ $\mathrm{ng} / \mathrm{mL}$ (Table 4). The Wald test was performed to attain an odds ratio in predicting severe DF for each variable. Out of the 4 variables, only lethargy and albumin of $<35 \mathrm{~g} / \mathrm{L}$ showed high odds ratios of significant values (lethargy OR 10.95 , 95\% CI 2.7144.22, $p=0.001$; albumin OR 5.72, 95\% CI 1.55-21.05, $p=0.009)$. Death is found to be associated with a higher median plasma PCT level of $3.6 \mathrm{ng} / \mathrm{mL}$ (range 3.2-4.0 $\mathrm{ng} / \mathrm{mL}$ ) while survivors had a lower median plasma PCT level of $0.28 \mathrm{ng} / \mathrm{mL}$ (range $0.17-0.57 \mathrm{ng} / \mathrm{mL})(p=0.021)$. There is no significant difference in plasma lactate $(p=$ $0.494)$ and plasma albumin $(p=0.165)$ levels between survivors and deceased subjects (Table 5).

\section{Receiver Operating Characteristic (ROC) Curve Analysis}

To further assess plasma PCT's specificity and sensitivity in predicting severe DF, a ROC curve analysis was conducted. When PCT $>0.3 \mathrm{ng} / \mathrm{mL}$ is combined with lethargy and albumin $<35 \mathrm{~g} / \mathrm{L}$, the AUROC is improved to 0.86 (95\% confidence interval 
[95\% CI]: 0.74-0.98). These parameters give a prediction of severe DF, making it a promising sensitivity of $73 \%$ and specificity of $85 \%$ in the

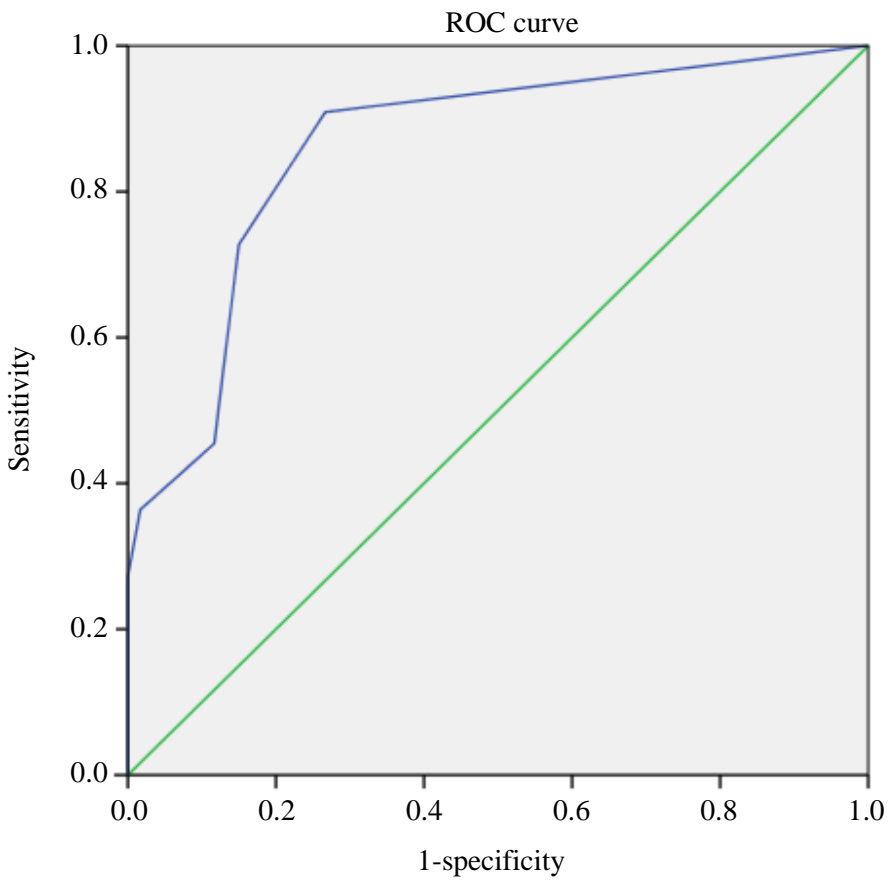

Diagonal segments are produced by ties.

Fig. 2: Receiver operating characteristic curve for lethargy, albumin $<35 \mathrm{~g} / \mathrm{L}$ and plasma procalcitonin $>0.3 \mathrm{ng} / \mathrm{mL}$. The AUROC was 0.86 (95\% confidence interval [95\% CI]: 0.74-0.98)

Table 3: Demographics, warning signs and clinical parameters between severe and non-severe groups

\begin{tabular}{llll}
\hline Characteristics & Non severe $(\mathrm{n}=117), \mathrm{n}(\%)$ & Severe $(\mathrm{n}=16), \mathrm{n}(\%)$ & $\mathrm{p}$-value \\
\hline Female & $49(42)$ & $7(44)$ & 0.277 \\
Defervescence phase & $36(30.8)$ & $9(56.3)$ & 0.043 \\
Warning signs & & & \\
Abdominal pain & $28(23.9)$ & $4(25)$ & 0.925 \\
Vomiting & $59(50.4)$ & $8(50)$ & 0.974 \\
Diarrhoea & $62(52.9)$ & $4(25)$ & 0.060 \\
Lethargy & $9(7.7)$ & $7(43.8)$ & 0.000 \\
Spontaneous bleeding & $9(7.7)$ & $3(18.8)$ & 0.159 \\
Tender hepatomegaly & $2(1.7)$ & $0(0)$ & 1.000 \\
Clinical fluid accumulation & $0(0)$ & $1(6.3)$ & 0.120 \\
Past medical history & $15(12.8)$ & $4(25)$ & 0.355 \\
Parameters & Median $(\mathrm{IQR})$ & $\mathrm{Median}(\mathrm{IQR})$ & $\mathrm{p}$-value \\
Age, years & $30(23-46)$ & $28(18-59)$ & 0.630 \\
Systolic blood pressure, $\mathrm{mmHg}$ & $120(107-129)$ & $94(84-106)$ & 0.000 \\
Diastolic blood pressure, $\mathrm{mmHg}$ & $76(67-82)$ & $56(49-65)$ & 0.000 \\
Pulse rate per minute & $88(76-98)$ & $92(88-101)$ & 0.275 \\
Temperature, ${ }^{\circ} \mathrm{C}$ & $38.2(37.6-38.8)$ & $37.8(37.1-38.3)$ & 0.069 \\
Respiratory rate per minute & $20(18-20)$ & $20(19-21)$ & 0.093 \\
\hline
\end{tabular}

Table 4: Multivariate analysis on significant parameters

\begin{tabular}{llll}
\hline Variable & OR & $95 \%$ CI & p-value \\
\hline Defervescence phase & 2.6 & $0.73-9.21$ & 0.138 \\
Lethargy & 10.95 & $2.71-44.22$ & 0.001 \\
Albumin $<35 \mathrm{~g} / \mathrm{L}$ & 5.72 & $1.55-21.05$ & 0.009 \\
Procalcitonin $>0.3 \mathrm{ng} / \mathrm{mL}$ & 1.36 & $0.39-4.7$ & 0.629 \\
\hline
\end{tabular}


Table 5: Plasma lactate and plasma procalcitonin levels in relation to outcome

\begin{tabular}{llll}
\hline Plasma parameter & Survival $(\mathrm{n}=131)$ & Death $(\mathrm{n}=2)$ & $\mathrm{p}$-value \\
\hline Albumin, $\mathrm{g} / \mathrm{L}$ & $38(34-41)$ & $19(17-21)$ & 0.165 \\
Lactate, $\mathrm{mmol} / \mathrm{L}$ & $1.63(1.21-2.22)$ & $1.34(1.26-1.42)$ & 0.494 \\
Procalcitonin, $\mathrm{ng} / \mathrm{mL}$ & $0.28(0.17-0.57)$ & $3.6(3.2-4.0)$ & 0.021 \\
\hline
\end{tabular}

\section{Discussion}

This study's subjects are mainly young to middleaged adults with a median age of 30 [IQR (23-46)] years. Involvement of this particular age group is possibly attributed to their active lifestyle outdoors, thus exposing them to vectors more so during the feeding period of the Aedes spp. mosquitoes. Being of a working population in an urbanised area in Selangor also puts this particular age group at risk of Dengue virus infection. A majority of recruits were Malays followed by Chinese, Indians and Aborigines - a finding that accords to the country's racial distribution. Foreigners with Dengue fever are underreported due to their limited access to healthcare in Malaysia.

A large fraction of subjects were admitted on median day 5 [IQR (3-6)] of illness in febrile phase which is the phase when patients are ill, requiring close observation and medical intervention. This is mostly observed in the non-severe group as the severe DF group mainly presented in defervescence $(p=0.043)$. Patients with severe DF were also more likely to present with lethargy (multivariate analysis $p=0.001$ ) on admission, advocating that this complaint or symptom should be taken seriously by the attending physician. We define lethargy as a state of energy insufficiency affecting Activities of Daily Living (ADL). Previous studies on prediction and prognostication of DF from signs and symptoms however did not show a significant relationship between lethargy and severe DF (Thein et al., 2013; Sreenivasan et al., 2018; Joshua et al., 2016).

Since lethargy is a subjective complaint, we suggest a classification model to grade lethargy according to severity. This grading system should be practical, simple, user-friendly and beneficial for clinical practice. We propose a scoring system or visual analogue scale to make this continuum of values more objective. With this grading system, emphasis may be put on extracting this particular symptom during the history-taking process.

The median hospital admission stay of 4 [IQR (3-5)] days duration confers with the disease course where at the end of the 4-day stay, patients have had gone into critical phase for $48 \mathrm{~h}$ and subsequently improved in recovery phase. On presentation, patients who presented with warning signs other than lethargy were not specific to severe or non-severe DF. This indicates that signs are non-specific and non-sensitive for predicting severe DF thus cannot be solely relied on for stratification purposes. This concurs with the World Health
Organization (WHO) 2009 guidelines into management of DF where the presence of warning signs is an indication for hospital admission (WHO, 2009).

In the $16(12 \%)$ patients with severe DF, 7 (44\%) had severe plasma leakage and $9(56 \%)$ had organ impairment of which $2(1.5 \%)$ succumbed. Patients with severe DF were found to have lower median systolic and diastolic blood pressures of $94 \mathrm{mmHg}$ and $56 \mathrm{mmHg}$ respectively ( $p=0.000)$ as compared to their non-severe counterparts. This is expected to happen in the severe spectrum of the disease due to inflammatory responses causing vasodilatation and plasma leakage from the capillaries.

As a result from the dynamic course of DF, 111 $(83.5 \%)$ subjects had thrombocytopenia as defined as platelets of less than $150 \times 10^{9} \mathrm{~L}$ on the day of admission, with $46(34.6 \%)$ of subjects having platelet counts of less than $50 \times 10^{9} / \mathrm{L}$. However, despite the high frequency of thrombocytopenia, only $12(9 \%)$ subjects had mucosal bleeds and none had major bleeds requiring platelet transfusion. This finding supports the 2015 Malaysian Clinical Practice Guidelines (CPG) on management of Dengue fever where prophylactic platelet transfusion is not recommended in Dengue fever with thrombocytopenia unless an invasive procedure or surgery is intended on a patient (CPG Malaysia, 2015).

Biochemically, plasma albumin of $<35 \mathrm{~g} / \mathrm{L}$ was found to be significantly associated with severe DF by both univariate and multivariate analyses. This is explained by the systemic inflammatory response causing reduced production, increased catabolism and third space loss (Ruot et al., 2000). Albumin is also used as a parameter in calculating the Acute Physiology and Chronic Health Evaluation (APACHE) III score to prognosticate critically ill patients, thus highlighting it as a significant negative acute phase reactant (Knaus, 2002). This finding correlates with a 2017 study conducted in New Delhi, India where low albumin had a significant value on DF survival outcome. Only 2 parameters reported significant values on multivariate analysis which are hypoalbuminemia $(p=0.013)$ and altered sensorium $(p=0.006)$. Both were identified as independent predictors of mortality for Dengue fever (Saroch et al., 2017). A published systematic review and meta-analysis in 2013 involving 198 studies also found a significant association between hypoalbuminemia and Dengue Shock Syndrome (Huy et al., 2013).

Lactate, on the other hand, shows no significant association with death as opposed to survival ( $p=$ 0.494), making it an unreliable prognostic marker. We acknowledge these results were different in comparison 
to a study conducted in Singapore where lactate proves to have better prognostic accuracy compared to baseline PCT (Phua et al., 2008). This contradictory correlation is probably due to our study's design where plasma lactate was taken only once on admission and at different times of the disease course.

The prediction of developing severe DF improves when PCT is combined with lethargy and plasma albumin of $<35 \mathrm{~g} / \mathrm{L}$ (AUC 0.86). AUROC analysis of these three parameters revealed a sensitivity of $73 \%$ and specificity of $85 \%$ in predicting severe dengue on the first day of admission to hospital.

\section{Conclusion}

While most patients presented on median day 5 (IQR 4-6) of illness, those with severe Dengue fever tend to present in defervescence phase and those with nonsevere Dengue fever in febrile phase. Lethargy - a state of lack of energy affecting Activities of Daily Living (ADL) - is a symptom that is often overlooked and dismissed but a significant presenting feature in severe DF on top of a plasma albumin of $<35 \mathrm{~g} / \mathrm{L}$. In our cohort, other warning signs were not significantly associated with severe DF, making warning signs a non-specific, non-sensitive and unreliable tool in classifying or prognosticating patients with DF. Laboratory parameters such as renal profile, electrolytes, AST and bilirubin showed no significant difference across board. PCT was raised to the $0.1-0.5 \mathrm{ng} / \mathrm{mL}$ range in both groups with no significant difference between the two. However PCT may be useful in the prognostication of DF where: (1) at a cut-off of $>0.3 \mathrm{ng} / \mathrm{mL}$, it predicts severe $\mathrm{DF}$ at a sensitivity level of $73 \%$ and specificity of $85 \%$ when combined with lethargy and albumin $<35 \mathrm{~g} / \mathrm{L}$; and (2) it is significantly associated with death when raised to a median of $3.6 \mathrm{ng} / \mathrm{mL}$. Lactate was similarly raised (median of 1.63 $\mathrm{mmol} / \mathrm{L}$ ) with no significant difference between both groups but unlike PCT, it is not associated with death.

Conclusively, higher median PCT value is seen in severe DF although no association is seen in multivariate analysis. However, when PCT is combined with lethargy and hypoalbuminemia, it is able to predict severe DF on day 1 of admission itself.

\section{Acknowledgement}

We thank everyone involved in this study for their contributions. We extend our gratitude to Internal Medicine team members, participants and staff of Selayang Hospital.

\section{Author's Contributions}

Huzairi Sani: Wrote the manuscript, involved in data collection and analysis.
Nada Syazana: Involved in data analysis and assessed the quality of studies.

Zahir Izuan: Involved in critical revision of the manuscript.

Mohamad Rodi Isa: Performed data analysis.

Muhammad Hanis: Involved in critical revision of the manuscript.

Muhamad Yazli: Supervising and oversight the project.

\section{Funding Information}

This is a self-funded clinical trial.

\section{Ethics}

The study protocol follows the principles governed by the World Medical Association Declaration of Helsinki. This study is approved by the National Medical Research Registry (NMRR) with the registration number NMRR17-1677-36506; as well as by the UiTM University Ethics Board [Reference number: 600-RMI (5/1/6)].

\section{References}

Becker, K.L., E.S. Nylén, J.C. White, B. Müller and R.H. Snider Jr., 2004. Clinical review 167: Procalcitonin and the calcitonin gene family of peptides in inflammation, infection and sepsis: A journey from calcitonin back to its precursors. J. Clin. Endocrinol. Metab., 89: 1512-1525.

DOI: $10.1210 /$ jc.2002-021444

Bur, R., S. Suwarto, W.D. Santoso and K. Harimurti, 2016. Serum lactate as predictor and diagnostic biomarker of plasma leakage in adult dengue patients. Universa Medicina.

DOI: 10.18051/UnivMed.2016.v35.213-221

Chen, C.M., K.S. Chan, H.C. Chao and C.C. Lai, 2016. Diagnostic performance of procalcitonin for bacteremia in patients with severe dengue infection in the intensive care unit. J. Infect., 73: 93-95. DOI: 10.1016/j.jinf.2016.03.013

CPG Malaysia, 2015. CPG Management of dengue infection in adults Malaysia. Academy of Medicine of Malaysia.

CPG Malaysia, 2016. Malaysia dengue incidence rate and case fatality rate.

CPG Malaysia, 2018. iDengue for community.

de Werra, I., C. Jaccard, S.B. Corradin, R. Chiolero and B. Yersin et al., 1997. Cytokines, nitrite/nitrate, soluble tumor necrosis factor receptors and procalcitonin concentrations: Comparisons in patients with septic shock, cardiogenic shock and bacterial pneumonia. Crit. Care Med., 25: 607-613. DOI: 10.1097/00003246-199704000-00009 
Giamarellos-Bourboulis, E.J., P. Giannopoulou, P. Grecka, D. Voros and K. Mandragos et al., 2004. Should procalcitonin be introduced in the diagnostic criteria for the systemic inflammatory response syndrome and sepsis? J. Crit. Care, 19: 152-157. DOI: 10.1016/j.jcrc.2004.07.001

Huy, N.T., T.V. Giang, D.H.D. Thuy, M. Kikuchi and T.T. Hien et al., 2013. Factors associated with dengue shock syndrome: A systematic review and meta-analysis. PLoS Negl. Trop. Dis., 7: e2412-e2412. DOI: 10.1371/journal.pntd.0002412

Joshua, G.X., T.L.T. Wong, Y.S. Leo, J. Pang and D.C. Lye, 2016. Identifying adult dengue patients at low risk for clinically significant bleeding. PLoS One.

Knaus, W.A., 2002. APACHE 1978-2001: The development of a quality assurance system based on prognosis. Arch. Surg., 1: 37-41.

DOI: 10.1001/archsurg.137.1.37

Leo, Y.S., V.C. Gan, E.L. Ng, Y. Hao and L.C. Ng et al., 2013. Utility of warning signs in guiding admission and predicting severe disease in adult dengue. BMC Infect. Dis., 13: 498-498.

DOI: $10.1186 / 1471-2334-13-498$

Lin, C.Y., C.H. Huang and Y.H. Chena, 2013. Classification of dengue: The clinical use of World Health Organization 2009 guideline. J. Med. Assoc., 112: 61-63. DOI: 10.1016/j.jfma.2012.06.005

Linscheid, P., D. Seboek, D.J. Schaer, H. Zulewski and U. Keller et al., 2004. Expression and secretion of procalcitonin and calcitonin gene-related peptide by adherent monocytes and by macrophage-activated adipocytes. Crit. Care Med., 32: 1715-1721. DOI: 10.1097/01.CCM.0000134404.63292.71

Mia, M.S., R.A. Begum, A.C. Erc, R.D.Z.R. Zain-alAbidin and J.J. Pereira et al., 2013. Trends of dengue infections in Malaysia, 2000-2010. Asian Pac. J. Trop. Med., 6: 462-466.

DOI: $10.1016 / \mathrm{S} 1995-7645(13) 60075-9$

Moya, F., A. Nieto and J.L. R-Candela, 1975. Calcitonin biosynthesis: Evidence for a precursor. Eur. J. Biochem., 55: 407-413. DOI: $10.1111 /$ j.1432-1033.1975.tb02176.x

Novotny, A., K. Emmanuel, E. Matevossian, M. Kriner and K. Ulm et al., 2007. Use of procalcitonin for early prediction of lethal outcome of postoperative sepsis. Am. J. Surg., 194: 35-39.

DOI: 10.1016/j.amjsurg.2006.10.026

Oberhoffer, M., W. Karzai, A. Meier-Hellmann, D. Bögel and J. Fabinder et al., 1999. Sensitivity and specificity of various markers of inflammation for the prediction of tumor necrosis factor- $\alpha$ and interleukin-6 in patients with sepsis. Crit. Care Med., 27: 1814-1818.

DOI: $10.1097 / 00003246-199909000-00018$
Phua, J., E.S.C. Koay and K.H. Lee, 2008. Lactate, procalcitonin and amino-terminal pro-B-type natriuretic peptide versus cytokine measurements and clinical severity scores for prognostication in septic shock. Shock, 29: 328-333.

DOI: 10.1097/SHK.0b013e318150716b

Ruot, B., D. Breuillé, F. Rambourdin, G. Bayle and P. Capitan et al., 2000. Synthesis rate of plasma albumin is a good indicator of liver albumin synthesis in sepsis. Am. J. Physiol. Endocrinol. Metabolism, 279: E244-E251. DOI: 10.1152/ajpendo.2000.279.2.E244

Saroch, A., V. Arya, N. Sinha, R.S. Taneja and P. Sahai et al., 2017. Clinical and laboratory factors associated with mortality in dengue. Trop. Doct., 47: 141-145. DOI: 10.1177/0049475517693993

Simmons, C.P., J.J. Farrar, N.V.V. Chau and B. Wills, 2012. Dengue. N. Engl. J. Med., 366: 1423-1432. DOI: $10.1056 / \mathrm{NEJMra1} 110265$

Singer, M., C.S. Deutschman and C.W. Seymour, 2016. The third international consensus definitions for sepsis and septic shock (Sepsis-3). JAMA, 315: 801-810. DOI: 10.1001/jama.2016.0287

Spoto, S., E. Cella, M. de Cesaris, L. Locorriere and S. Mazzaroppi et al., 2018. Procalcitonin and $\mathrm{mr}$ proadrenomedullin combination with sofa and qsofa scores for sepsis diagnosis and prognosis: A diagnostic algorithm. Shock, 50: 44-52.

DOI: 10.1097/SHK.0000000000001023

Sreenivasan, P., S. Geetha and K. Sasikala, 2018. Development of a prognostic prediction model to determine severe dengue in children. Indian $\mathrm{J}$. Pediatr, 85: 433-439. DOI: $10.1007 / \mathrm{s} 12098-017-2591-y$

Thanachartwet, V., V. Desakorn, D. Sahassananda, A. Jittmittraphap and N. Oer-Areemitr et al., 2016. Serum procalcitonin and peripheral venous lactate for predicting dengue shock and/or organ failure: A prospective observational study. PLoS Negl. Trop. Dis., 10: e0004961.

DOI: 10.1371/journal.pntd.0004961

Thein, T.L., V.C. Gan, D.C. Lye, C.F. Yung and Y.S. Leo et al., 2013. Utilities and limitations of the world health organization 2009 warning signs for adult dengue severity. PLoS Negl. Trop. Dis., 7: e2023. DOI: 10.1371/journal.pntd.0002023

WHO, 2009. Dengue: Guidelines for diagnosis, treatment, prevention and control. 